\title{
CURVE SPACE: CLASSIFYING CURVES ON SURFACES
}

\author{
XIN LI*, XIANFENG GU*, AND HONG QIN*
}

\begin{abstract}
We design signatures for curves defined on genus zero surfaces. The signature classifies curves according to the conformal geometry of the given curves and their embedded surface. Based on Teichmüller theory, our signature describes not only the curve shape but also the intrinsic relationship between the curve and its embedded surface. Furthermore, the signature metric is stable, it is close to identity between surfaces sharing similar Riemannian geometry metrics. Based on this, we propose a surface matching framework: first, with curve signatures, we match the partitioning of two surfaces defined by simple closed curves on them; second, the segmented subregions are pairwisely matched and then compared on canonical planar domains.
\end{abstract}

1. Introduction. Shape analysis and shape comparison are fundamental problems in computer vision, graphics and modeling fields with many important applications. Lots of $2 \mathrm{D}$ and $3 \mathrm{D}$ shape analysis techniques have been developed in the past couple of decades, most of which are based on comparing curvature or spacial positions of the points on the curve.

A complete different way is to consider all the closed curves on the surface. The curve space on surface conveys rich geometric information of the surface itself and is easy to process. The philosophy of analyzing shapes by their associated curve spaces has deep root in algebraic topology [8], infinite dimensional Morse theory [18] and Teichmüller space theory in complex geometry [31].

Suppose $M$ is a surface (a 2-manifold), a closed curve on $M$ is a map

$$
\gamma:[0,1] \rightarrow M, \gamma(0)=\gamma(1)
$$

We define the set of all simple closed curves on $M$ as curve space and denote it as $\Omega(M)$. In algebraic topology, $\Omega(M)$ is classified by homotopy relation,

$$
\gamma_{0} \sim \gamma_{1}: \exists F:[0,1] \times[0,1],\left.F(\cdot)\right|_{0}=\gamma_{0},\left.F(\cdot)\right|_{1}=\gamma_{1},
$$

then the quotient space $\Omega(M) / \sim$ forms a group $\pi_{1}(M)$, the addition of $\pi_{1}(M)$ is the concatenation of the curves. All the topological information of $M$ is reflected by the algebraic structure of its fundamental group $\pi_{1}(M)$.

Milnor pointed out the $\Omega(M)$ is an infinite dimensional manifold, a nature curve length function is introduced

$$
f: \Omega(M) \rightarrow \mathbb{R}, f(\gamma)=\int_{0}^{1}<\dot{r}, \dot{r}>d t
$$

*Stony Brook University, Stony Brook, NY 11794, USA. E-mail: \{xinli, gu, qin\}@cs.sunysb.edu 
where $\langle\cdot, \cdot\rangle$ is the inner product in $\mathbb{R}^{3}$. It is shown that $f$ is a Morse function, its critical points are geodesics. By analyzing the local structures of the critical points, the topology of $\Omega(M)$ can be obtained. Furthermore, the topology of $\Omega(M)$ determines the topology of $M$. In differential geometry, locally, the surface shape is completely determined by the curves in the neighborhood. For example, the curvatures of the sectional curves through one point specify the principle curvatures of that point.

Our current research follows this philosophy but is based on Teichmüller space theory. Suppose $M_{1}$ and $M_{2}$ are two surfaces, $\phi: M_{1} \rightarrow M_{2}$ is a diffeomorphism ${ }^{1}$ between them. Then any curve $\Gamma_{1} \in \Omega\left(M_{1}\right)$ will be mapped to a curve in $\Omega\left(M_{2}\right)$ by $\Gamma_{2}=\phi \circ \Gamma_{1}$. Therefore $\phi$ induces a one to one map $\phi^{*}$ from $\Omega\left(M_{1}\right)$ to $\Omega\left(M_{2}\right)$ by $\phi^{*}: \Omega\left(M_{1}\right) \rightarrow \Omega\left(M_{2}\right)$. Instead of studying $\phi$, we can analyze relations between surfaces by studying $\phi^{*} M$.

Furthermore, we map the curve space $\Omega(M)$ to a canonical Lie group $\operatorname{Diff}\left(S^{1}\right)$, where $\operatorname{Diff}\left(S^{1}\right)$ denotes the group of all diffeomorphism from the unit circle $S^{1}$ to itself. We denote this map as $g_{i}$, with $g_{1}: \Omega\left(M_{1}\right) \rightarrow \operatorname{Diff}\left(S^{1}\right)$ and $g_{2}: \Omega\left(M_{2}\right) \rightarrow$ $\operatorname{Diff}\left(S^{1}\right)$. $\Phi^{*}: \Omega\left(M_{1}\right) \rightarrow \Omega\left(M_{2}\right)$ induces a mapping from $\operatorname{Diff}\left(S^{1}\right)$ to itself by $\bar{\Phi}:=g_{2} \circ \Phi^{*} \circ g_{1}^{-1}$.

The process discussed above is summarized as the following diagram:

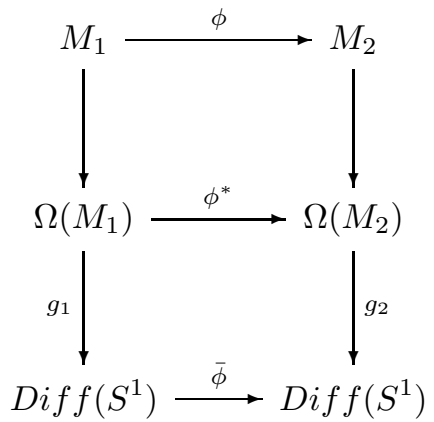

This diagram demonstrates our methodology: three mappings $\Phi, \Phi^{*}, \bar{\Phi}$ are closely related. In fact, any one of them determines the other two. In other words, for the purpose of studying surfaces $M_{1}, M_{2}$ and the maps $\Phi$ among them, we can study their curve spaces $\Omega\left(M_{1}\right), \Omega\left(M_{2}\right)$, signatures of the curves Diff $\left(S^{1}\right)$, and the mappings $\Phi^{*}, \bar{\Phi}$ among them. The following theoretic results clarify the intuition.

Theorem 1. $M$ is a metric oriented surface, the curve space $\Omega(M)$ and Diff $\left(S^{1}\right)$ are equipped with $L^{2}$ metric, the map $\Psi$ from its curve space $\Omega(M)$ to $\operatorname{Diff}\left(S^{1}\right)$ is a homeomorphism.

Therefore, to measure the distance between two curves on a surface, the distance between two signatures in $\operatorname{Diff}\left(S^{1}\right)$ is sufficient.

\footnotetext{
${ }^{1} \mathrm{~A}$ function is differentiable and has a differentiable inverse.
} 
Theorem 2. Suppose $M_{1}$ and $M_{2}$ are two oriented metric surfaces, $\phi$ is a conformal map if and only if $\bar{\Phi}$ is the identity map of Diff $\left(S^{1}\right)$.

The mapping from $\Phi$ to $\bar{\Phi}, F: \Phi \rightarrow \bar{\Phi}$ discovers a lot of geometric information about $M_{1}$ and $M_{2}$. It is highly possible that, by choosing appropriate metrics, $F$ is continuous. The kernel of $F$ is all the conformal mapping between $M_{1}$ and $M_{2}$.

\section{Contributions:}

1 In theoretical aspect, we design curve signatures for curves on surfaces, which can be used to study curves on the same surface or on different but similar surfaces. (Section 2)

2 With the mapping $\bar{\phi}$, we can study the mapping $\Phi$ between surfaces, more specifically, surface matchings are guided by feature curve matchings. (Section 3)

Curve space on surfaces contains much richer information compared to planar curve space. Planar curve space theory [27] characterizes the curves themselves, whereas our curve space on surface emphasizes both the curves and their relations with the embedded surfaces. In other words, our signatures classify and compare curves based on how they segment the embedded surface. The curve space on surface can be applied for geometric processing of surfaces such as shape comparison and registration. Planar curve space can be treated as a special case of our general surface curve space. Although in this paper, we focus on genus zero surfaces only, the theoretic framework can be generalized to arbitrary surfaces.

1.1. Related work. In computer vision/graphics fields, existing effective curve matching and comparison methods focused on properties of planar curve itself $[5,32$, $2,26,30,7,15,23,3,19]$.

Recent research on conformal geometry opens a new way to study curve and shape matching. [27] modeled the planar simple closed smooth curves by diffeomorphisms from a circle to itself via conformal mapping and proved the space of all such curves modulo scaling and transformation is isomorphic to the diffeomorphism group of the circle quotient Möbius transformations group restricted on the circle. Conformal geometry was also applied by for surface classification and matching $[13,10]$.

Current available techniques for surface matching and comparison typically fall into several categories. A large number of shape descriptors attempt to label shapes using histograms collecting specific global properties of the underlying object. Ankerst et al. [1] defined the histogram of the volume distributed on concentric shells and sectors, and used a quadratic form to measure distance between two objects. Osada et al. [22] used the probability of distances between two randomly sampled points from the surface as its shape descriptor. Ohbuchi et al. [21] presented an inertial principal axis histogram about many sampled axes for shape comparison. On each 
axis, the moment of the inertia, the average distance and its variance from surface points to the axis constitute the histogram. Kazhdan et al. [17] introduced a reflective symmetry descriptor as a surface analysis and rigid-body alignment tool, which is a histogram consisting of distances from surface points to planes passing through the mass center. Gal et al. [9] designed a histogram that comprises a so called local diameter function and the $D 2$ functions introduced in [22] to arrive at a pose-oblivious descriptor. Another type of descriptors usually comes from signal decomposition methods, Saupe and Vranic [25] introduced a ray-based spherical harmonic descriptor. Kazhdan et al. [16] also used the spherical harmonics but they worked on voxel grids. Novotni and Klein [20] proposed to use a more generalized 3D Zernike function as the base function. Reuter et al. [24] designed the descriptor using the Laplacian shape spectrum. The third type of descriptors relies on the skeleton or the topology structure of the surface. Sundar et al. [29] designed a skeleton descriptor which converts the object to its volume skeleton and they matched the skeleton graphs using methods introduced in [28]. Hilaga et al. [14] and Biasotti et al. [4] used Reed graph as the shape descriptor. Dey et al. [6] compared shapes by analyzing flow and their critical points on surface. The vast majority of the existing work try to compare the objects in a global sense relying on their geometry information, without any involvement of semantic feature curves. In contrast, our method tackles the shape comparison in a divide-and-conquer way so that the comparison between complex objects can be greatly simplified, while at the same time, with the help of feature alignments, the matching can be more flexible and application-specific. Furthermore, while we are capable of quantitatively identify the overall, global shape difference, one key feature of our curve-centric comparison is to offer the local shape variation and its distribution in order to facilitate shape registration, segmentation, and analysis.

2. Signatures in Curve Space. This section outlines our theoretical results on how to compute curve signatures for curves defined on a surface. Simple closed planar curves can be represented as a diffeomorphism from unit circle to itself [27] up to the scaling and translation. For spatial curves defined on a surface, we also use a diffeomorphism ${ }^{2}$ from the unit circle to itself to represent it. This diffeomorphism represents the spatial curve on surface uniquely up to a Möbius transformation. By removing the Möbius ambiguity using some special markers, we obtain the signatures corresponding to the curves bijectively.

2.1. Theory and Algorithm Overview. Given a simple closed curve $\Gamma$ on a genus zero surface $M$, the central idea to compute its signature is illustrated in Figure 1(a), (b) and (c).

\footnotetext{
${ }^{2}$ Such a diffeomorphism can be viewed as a real periodic function from $[0,2 \pi]$ to itself.
} 


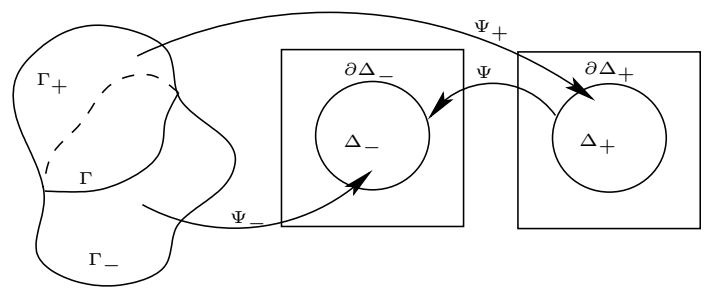

(a) Closed, genus zero surface.

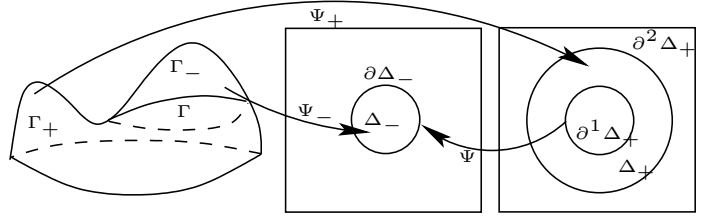

(b) Open, genus zero surface.

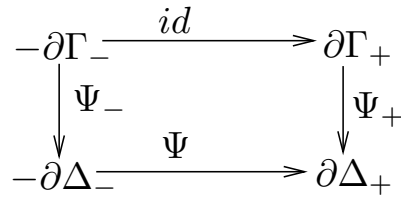

id = identity map

(c) Curve signature from boundary mappings.

FIG. 1. Curves as equivalence classes of diffeomorphisms.

Case 1: If $M$ is closed, as shown in Figure 1(a), then $\Gamma$ partitions $M$ into two components $\Gamma_{+}, \Gamma_{-}$, both are topological disks and can be conformally mapped onto planar unit disks $\Delta_{+}, \Delta_{-}$by $\Psi_{+}, \Psi_{-}$. $\Gamma$ is the boundary of $\Gamma_{+}$and $\Gamma_{-}$, denoted by $\partial \Gamma_{+}=\Gamma$ and $\partial \Gamma_{-}=-\Gamma$, and is mapped to the disk boundary, which is the unit circle $\Delta=\partial \Delta_{+}=-\partial \Delta_{-}$. The mapping induced by $\Psi_{+}$and $\Psi_{-}$on the boundaries $\partial \Delta_{+}$ and $\partial \Delta_{-}$is a diffeomorphism. This diffeomorphism $\Psi: \partial \Delta_{+} \rightarrow \partial \Delta_{-}$is the signature of $\Gamma$.

Case 2: If $M$ is open, as shown in Figure 1(b), then $\Gamma$ partitions $M$ into a topological disk $\Gamma_{-}$and a topological annulus $\Gamma_{+} . \Gamma_{-}$can be conformally mapped onto a unit disk $\Delta_{-}$, while $\Gamma_{+}$can be conformally mapped onto an annulus $\Delta_{+}$with unit inner radius. We denote such annulus with unit inner radius as canonical annulus, the inner boundary of $\Delta_{+}$as $\partial^{1} \Delta_{+}$, and use the diffeomorphism $\Psi: \partial \Delta_{-} \rightarrow \partial^{1} \Delta_{+}$ as the signature of $\Gamma$. One example is shown in Figure 2, the blue curve in genus zero open surface David head model (a) has its signature shown in (b).

In [27], Sharon and Mumford used Teichmüller theory to prove that any simple closed planar curve can be represented with such a diffeomorphism from a unit circle to itself uniquely up to scaling and translation. In this paper, we generalize this idea to arbitrary genus zero surface using Riemann surface theory. 


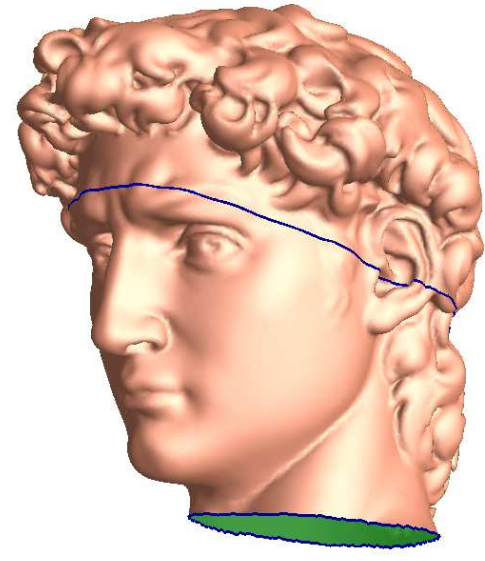

(a) Blue curve on david head surface.

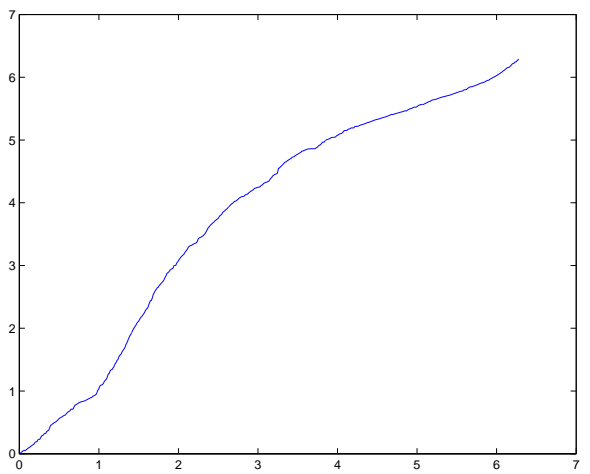

(b) Signature of the blue curve.

FIG. 2. Curve on David Head surface and its signature.

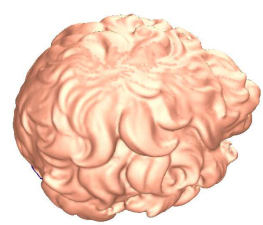

(a) Upper patch of David Head Surface

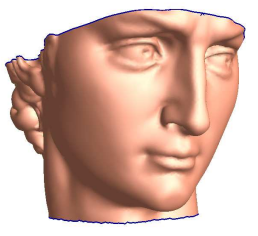

(e) Bottom patch of David Head Surface

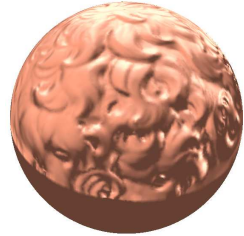

(b) Patch conformally mapped to sphere

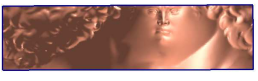

(f) Patch conformally mapped to rectangle

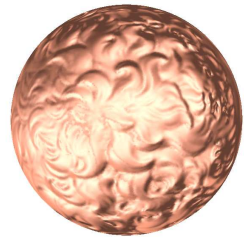

(c) Patch conformally mapped to disk

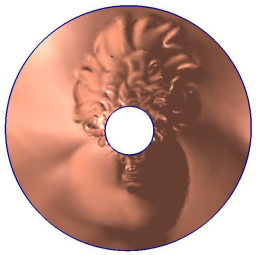

(g) Patch conformally mapped to annulus

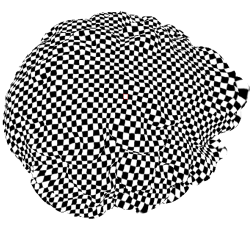

(d) Patch conformal

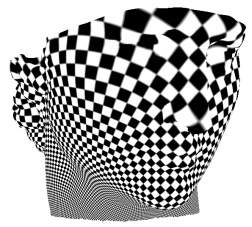

(h) Patch conformal texture mapping

Fig. 3. Conformal Mappings of SubPatches.

In technical essence, we compute the conformal mapping for each component segmented by the curve, and take the boundary mappings $\Psi$ as shown in Figure 1(c) as the signature. Some landmarks and constraints are used to eliminate the so-called Möbius ambiguity.

2.2. Conformal Map from an Open Genus-zero Surface to a Disk. We seek a conformal map $\Phi$ from a disk-like surface $M$ to a unit disk. The map does exist according to Riemann mapping theory. Extensive relevant work has been done on finding a good parameterization for disk-like surfaces. However, complete conformality is usually not guaranteed. Based on the fact that the harmonic map from a closed 
genus zero surface to a sphere is also conformal, we use the double covering technique [13] to convert an open surface to a closed one, and reduce computing $\Phi$ to computing a harmonic map from double covering of $M$ onto a sphere(Section 2.3).

For an open surface $M$, we compute the double covering of $M$ and then compute its harmonic mapping onto a sphere. Due to the exact symmetric property of double covering, the boundary $\partial M$ is harmonically mapped onto the equator of the sphere and $M$ is conformally mapped onto a hemisphere. Then we compose a stereo graphic projection to get a conformal map from $M$ to the unit disk. The procedure computing conformal map from topological disk in the previous example(Figure 2) onto the unit disk is shown in Figure 3(a)-(d).

\subsection{Conformal Map from a Closed Genus-zero Surface to a Sphere.}

To compute a conformal map $\Phi$ from a closed genus-zero surface $M$ to a sphere, we initiate a map between them and minimize the harmonic energy by diffusing the heatflow on the sphere surface. This process is introduced and proved to converge to a harmonic/conformal map [11].

The process is as follows:

1. Compute the normal vector for each triangle face. For each vertex, compute its normal $\vec{n}(v)$ as the weighed sum of normals on the adjacent faces weighed by their areas. Then set the initial map as the Gauss map: $\Phi(v)=\vec{n}(v)$.

2. Compute Laplace-Beltrami operator at each vertex: $\Delta(v)=\sum_{[u, v] \in M} w_{u, v}$ $(\Phi(v)-\Phi(u))$, the weight $w_{u, v}$ associated with edge $[u, v]$ is the well known harmonic weight, calculated as $w_{u, v}=\frac{1}{2}\left(\cot \left(\alpha_{u, v}\right)+\cot \left(\beta_{u, v}\right)\right)$, where $\alpha_{u, v}$ and $\beta_{u, v}$ are two angles opposite to the edge $[u, v]$ in the two triangles sharing the edge.

3. Project Laplacian $\Delta(v)$ onto the tangent space of $\Phi(v)$,

$$
\Delta_{s^{2}}(v)=\Delta(v)-(\Delta(v) \cdot \Phi(v)) \Phi(v) .
$$

4. Update the map $\Phi, \Phi(v)=\Phi(v)-\epsilon \Delta_{S^{2}}(v)$ where $\epsilon$ is a small constant to assure the numerical stability. In our experiment we set $\epsilon=0.1$.

5. Normalize the map $\Phi$ by

$$
\Phi(v)=\frac{\Phi(v)-\mathbf{c}}{|\Phi(v)-\mathbf{c}|}
$$

where $\mathbf{c}$ is the weighted mass center: $\mathbf{c}=\frac{\sum_{v} k_{v} \Phi(v)}{\sum_{v} k_{v}}$ where $k_{v}$ is the summation of the areas of all faces adjacent to vertex $v$.

6. Repeat step 2 through 5 until it converges.

2.4. Conformal Map from a Topological Annulus to a Canonical Annulus. For curves on an open genus-zero surface, we need to compute a conformal 
map $\Phi$ from a topological annulus $M$ (with $\partial M=\Gamma_{1}-\Gamma_{2}$ where $\Gamma_{1}$ and $\Gamma_{2}$ are two boundaries) to a canonical planar annulus. First, we double-cover the surface to get a closed genus-one surface; next we compute a conformal map from a closed genusone surface onto a rectangle planar domain by integrating a holomorphic 1-form [13] which describes two vector fields perpendicular to each other everywhere on surface; finally, we compose the conformal map from the rectangle to the canonical annulus using $e^{\frac{2 \pi}{b} z}$ to get the $\phi$.

The algorithmic flow is detailed as follows:

1. Double-cover $M$ to a closed genus one surface $\bar{M}$.

2. Compute a holomorphic 1-form basis of $\bar{M}$ by using the method introduced in [12]. Denote the basis as $\bar{\omega}$.

3. For an arbitrary path $\tau$ connecting $\Gamma_{0}$ and $\Gamma_{1}$, compute a holomorphic 1-form $\omega$ such that $\int_{\Gamma_{0}} \omega=1, \omega=\frac{1}{\int_{\Gamma_{0}} \bar{\omega}} \bar{\omega}$.

4. Trace a vertical trajectory $r$ of $\omega$, such that $r$ is an integration curve of $\omega$ along imaginary direction. Namely, $r$ is iso- $u$ in the $(u, v)$ domain.

5. Slice $M$ along $r$ to get a fundamental domain $\widetilde{M}$, by integrating $\omega$, where $\widetilde{M}$ is conformally mapped to a rectangle on the plane.

6. Conformally map the rectangle to an annulus with unit inner radius by $e^{\frac{2 \pi}{b}}$.

The procedure computing conformal map from a topological annulus in the previous example(Figure 2) onto the canonical annulus is shown in Figure 3(e)-(h).

2.5. Eliminating the Möbius Ambiguity. Conformal mappings between surfaces are not unique; e.g., all conformal mappings from a unit disk $D^{2}$ to itself form a Möbius group, with the form: $\tau: z \rightarrow w, w=e^{i \theta} \frac{z-z_{0}}{1-z_{0} z}, z, z_{0} \in \mathbb{C}, \theta \in[0,2 \pi)$, where $z_{0}$ is a constant point, $\theta$ is a constant angle. All such $\tau$ form a 3 real dimensional group. Two mappings from a topological disk to a unit disk differ by a Möbius transformation, this ambiguity affects the signature and has to be eliminated via certain extra constraints.

For closed genus-zero surfaces, we first fix a marker point $p$ on the surface and define a tangent direction $\vec{t}_{p}$ going out from $p$. A closed curve $\Gamma$ separates $M$ into two disk-topology patches, the patch containing $p$ is denoted as $\Gamma_{+}$. We require that $\Psi_{+}$ maps $p$ onto the origin, and $\vec{t}_{p}$ onto the positive x-axis direction. These constraints uniquely determine $\Psi_{+}$.

For open genus-zero surfaces, we fix the marker $p$ on the boundary. $\Psi_{+}$maps $\Gamma_{+}$ to $\Delta_{+}$, where $\Delta_{+}$is a canonical annulus with unit inner radius. The outer radius of $\Delta_{+}$is denoted as $R$, which is uniquely determined by the surface $\Gamma_{+}$. Furthermore, we require that $\Psi_{+}(p)=R$. Such $\Psi_{+}$uniquely exists.

Through the above construction pipeline, every closed curve $\Gamma \in \Omega(M)$ corresponds to a diffeomorphism $\Psi \in \operatorname{Diff}\left(S^{1}\right)$. $\Gamma$ corresponds to two signatures 
$\Psi_{1}, \Psi_{2}$ if and only if there exists a Mobsüs transformation $\tau: D^{2} \rightarrow D^{2}$, such that $\Psi_{2} \circ \Psi_{1}^{-1}=\left.\tau\right|_{\partial D^{2}}$. The above equation defines an equivalence relation $\sim \operatorname{in} \operatorname{Diff}\left(S^{1}\right)$. We claim that the mapping $\Psi: \Omega(M) \rightarrow \operatorname{Diff}\left(S^{1}\right) / \sim$ is an one-to-one map. With appropriate metrics on $\Omega(M)$ and $\operatorname{Diff}\left(S^{1}\right)$, it is a homeomorphism. In other words, each closed curve on $M$ corresponds to an equivalence class of diffeomorphisms from the unit circle to itself.

In some scenarios, we might want to completely eliminate the ambiguity of signatures. For this purpose, we can further eliminate Möbius ambiguity using more markers. To uniquely reconstruct a curve, $\Psi$ and three markers are sufficient for the closed genus-zero surfaces while for the open genus-zero surfaces, $\Psi$ and two markers are sufficient.

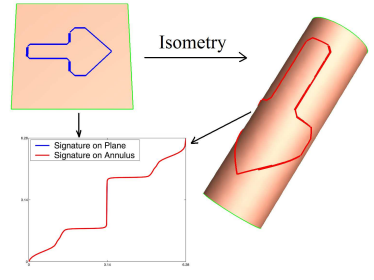

(1)

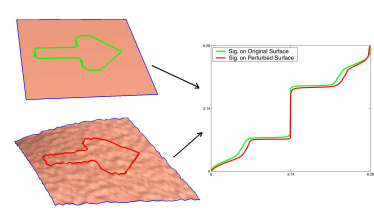

$(2)$

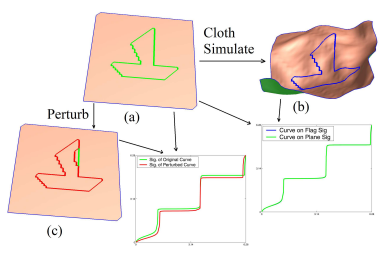

(3)

FIG. 4. The stability of curve signatures under isometry, perturbation and bending of embedded surfaces.

2.6. Distances between Curves. For a genus-zero surface $M$, we create signatures for curves defined on $M$. The deviation between two curves can be measured by the distance between their signatures using Weil-Peterson metric on Diff $\left(S^{1}\right)$ as introduced in [27].

If surfaces $M_{1}$ and $M_{2}$ are with similar Riemannian geometries in $\mathbb{R}^{3}$, then there exists a diffeomorphism $\phi: M_{1} \rightarrow M_{2}$ close to an isometry, the induced map $\bar{\phi}$ between the signatures is close to the identity map from $\operatorname{Diff}\left(S^{1}\right)$ to itself. In other words, if the curve $\Gamma_{1} \subset M_{1}$ corresponds to $\Gamma_{2} \subset M_{2}$ with $\Gamma_{2}=\phi\left(\Gamma_{1}\right)$, then $\Gamma_{1}$ and $\Gamma_{2}$ have similar signatures. Hence, the signatures of curves have a property of strong stability under the Riemannian metric perturbation of their embedded surfaces and can be used to analyze curves on different surfaces as a robust tool.

Figure 4 demonstrates the stability of the signatures. All the curves and their corresponding signatures are drawn in the same color. Note that the signature is a diffeomorphism from a circle to itself, thus it can be considered as a periodic real function from $[0,2 \pi)$ to $[0,2 \pi)$, and only one period is shown in our figures. In (1), a planar rectangle is isometrically deformed to a cylinder, our computation shows that the corresponding curves have exactly the same signatures. In (2), the planar 
rectangle is perturbed about $6 \%$ in $z$ direction, and about $1 \%$ in $x, y$ directions, signatures of the corresponding curves are very close to each other. In (3), the planar surface in $(a)$ is simulated as cloth and deforms as shown in (b), namely, it allows large bending but little stretching, the signatures of the corresponding curves are also almost identical(i.e., undistinguishable); also, the curve on surface in $(a)$ is perturbed a little and shown in red curve in $(c)$, the signature perturbs little.

Therefore, curves on different surfaces, which are close to each other in terms of geometry or differ by a near-isometric mapping, can be robustly and accurately compared and analyzed using their signatures.

3. Surface Matching. Based on the analysis of curve space, we design our surface matching framework for curve alignment, surface registration, and shape comparison.

3.1. Feature Alignment for Surface Segmentation and Matching. We now decompose the entire surface comparison problem into two sub-tasks: (1) segmenting a surface via a set of feature curves and their alignment; (2) matching boundary curves and surface patch interiors. The general framework is as follows.

Assume $M_{1}$ and $M_{2}$ are the two surfaces to be matched and compared, if they share similar geometries, meaning there exists a mapping $\phi: M_{1} \rightarrow M_{2}, \phi$ is close to an isometry, then

1. Extract a set of feature curves $\left\{\Gamma_{1}^{1}, \Gamma_{2}^{1}, \cdots, \Gamma_{n}^{1}\right\}$ on $M_{1}$, which can be either marked by users as certain meaningful features, or automatically computed based on geometric information of $M_{1}$ such as the extremals of the principal curvatures along the corresponding principal directions.

2. Compute the curve signatures of $\Gamma_{i}^{1}$ on $M_{1}$ using algorithms introduced in Section 2, and obtain the signature set $\left\{\Psi_{1}, \Psi_{2}, \cdots, \Psi_{n}\right\}$.

3. Compute the curve set $\left\{\Gamma_{1}^{2}, \Gamma_{2}^{2}, \cdots, \Gamma_{n}^{2}\right\}$, such that the curve signature of $\Gamma_{i}^{2}$ equals to $\Psi_{i}$.

4. The curve set $\left\{\Gamma_{i}^{k}\right\}$ segment $M_{k}$ to several connected components $\left\{c_{1}^{k}, c_{2}^{k} \cdots\right.$, $\left.c_{m}^{k}\right\}, k=1,2$, such that the boundaries of $c_{i}^{1}$ correspond to the boundaries of $c_{i}^{2}$.

5. Match $c_{i}^{1}$ with $c_{i}^{2}$ pairwise. $c_{i}^{1}$ and $c_{i}^{2}$ are conformally mapped to the canonical planar domains(done as a by-product from the process of computing signatures). We reduce the mapping between $3 \mathrm{D}$ surface $c_{i}^{1}$ and $c_{i}^{2}$ to the matching on their 2D planar domains.

From the theoretical perspective, the entire algorithmic pipeline can be formulated as the problem of solving $\phi: M_{1} \rightarrow M_{2}$ from knowing $\bar{\phi}=i d: \operatorname{Diff}\left(S^{1}\right) \rightarrow \operatorname{Diff}\left(S^{1}\right)$, then the resultant $\phi$ is the one closest to an isometry. $\phi$ is the best diffeomorphism for the surface registration between $M_{1}$ and $M_{2}$. 
In practice, in Step 3 above, users may prefer to label the meaningful feature curve set $\left\{\Gamma_{1}^{2}, \Gamma_{2}^{2}, \cdots, \Gamma_{n}^{2}\right\}$ on $M_{2}$. Then we compute their signatures and by comparing the signatures, we find an one-to-one matching between these two sets of feature curves. Then following Steps 4 and 5, the matching $\phi$ can be constructed in the similar way. Because the signatures depend on the curves continuously and stably, small deviations of the labeled feature curves will have no or very little affect on the final comparison result as far as the surface comparison is concerned.

3.2. Surface Comparison in $2 \mathrm{D}$ Planar Canonical Domains. When all feature curves are matched, we segment the surfaces into several patches, each of which is conformally mapped onto a canonical planar domain, then we reduce the 3D surface comparison task to an much easier 2D matching problem on the planar domain, which can possibly be solved with many existing techniques. A possible technique to solve such a problem is to use the conformal representation [10], which consists of two functions $(\lambda(u, v), H(u, v))$ defined on canonical domains, where $\lambda$ is called conformal factor, representing the area stretching of the mapping from the original surface to the planar domain and $H$ is the mean curvature implying the bending information of the surface. In our experiments, we normalize the original surface and then compute its conformal factor of each vertex by dividing its one-ring-neighbor area on the surface by its counterpart on the planar domain.

There are several advantages to measure surface patch difference using conformal representation. First, the conformal representation is complete in the sense that it allows us to fully reconstruct the original surface from the representation, which is guaranteed by the following theorem.

Theorem 3 (Conformal Representation). If a surface $S(u, v)$ is parameterized by some conformal parameter $(u, v)$ on a domain $D$, then the conformal factor function $\lambda(u, v)$ and mean curvature function $H(u, v)$ defined on $D$ satisfy the Gauss and Codazzi equation. If $\lambda(u, v)$ and $H(u, v)$ are given, along with the boundary condition $S(u, v) \mid \partial D$, then $S(u, v)$ can be uniquely reconstructed.

Second, according to [10], conformal representation stably represents the geometry distance between surfaces in $\mathbb{R}^{3}$; the perturbation in geometry leads to stable and continuous perturbation in their conformal representations.

Third, as a by-product, the computation process of curve signatures has already computed conformal maps from most 3D patches to the planar domains, so the surface matching based on these mappings can be done without further computation cost.

The matching energy between two corresponding surface patches $M_{0}$ and $M_{1}$ is defined on their common canonical planar domains $D: E=\int_{(u, v) \in D} \| \lambda_{0}(u, v)-$ $\lambda_{1}(u, v)\left\|^{2} d u d v+\int_{(u, v) \in D}\right\| H_{0}(u, v)-H_{1}(u, v) \|^{2} d u d v$.

Figure 5 shows an example on how to make use of conformal representation for 


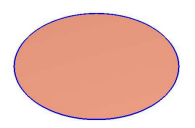

(a)

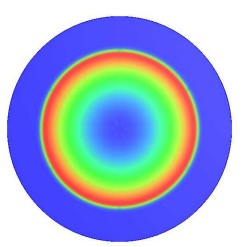

(d)

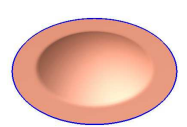

(b)

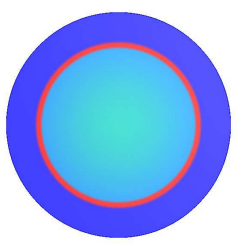

(e)

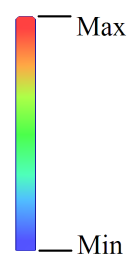

(c)

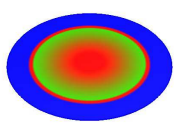

(f)

FIG. 5. Conformal representation. (a) and (b) are surfaces to be compared, (c) is the color scheme we use in this paper, (d) and (e) are conformal factor and mean curvature of (b) drawn in $2 D$ planar domain, and $(f)$ is the conformal representation difference distribution between (a) and (b).

surface comparison. A unit disk planar surface $M_{0}$ as shown in (a) is compared with a center-bulb surface $M_{1}$ shown in (b). The conformal factor and mean curvature of planar surface is constant everywhere; the conformal factor and mean curvature of surface (b) in 2D planar domain are color coded and shown in (d) and (e); the deviation $d(u, v)=\left(\lambda_{0}(u, v)-\lambda_{1}(u, v)\right)^{2}+\left(H_{0}(u, v)-H_{1}(u, v)\right)^{2}$ between the matched surfaces are color-coded in surface $M_{0}$ and shown in (f).

\section{Experimental results.}

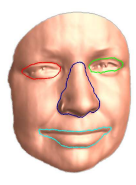

(a) $f_{0}$

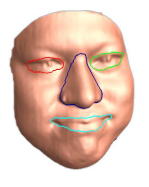

(b) $f_{1}$

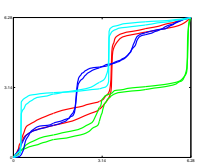

(c)

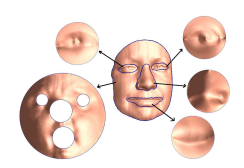

(d)

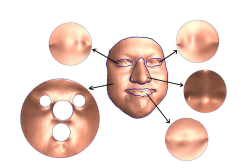

(e)

FIG. 6. Curves on faces((a),(b)), their signatures(c), and the segmentations for the matching purpose $((d),(e))$.

4.1. Human Faces. To illustrate our framework, we firstly present a human face matching example. Two human faces, $f_{0}$ (female) and $f_{1}$ (male), as shown in Figure 6 (a) and (b), are compared by aligning feature curves enclosing eyes, noses and mouths. Assuming that the geometries of human faces are similar, namely, there exist mappings $\Phi: f_{0} \rightarrow f_{1}$ that are close to isometry, we manually label on each face 
four feature curves and compute their signatures. The curves and their signatures are highlighted with the same color. For example, curves enclosing the right eyes and their signatures are colored in red. As shown in Figure 6 (c), signatures with the same color are quite similar to each other.

The experiment shows that similar feature curves on two faces have similar signatures, while different feature curves on the faces have dramatic different signatures. Therefore, the curve signature is a reliable tool to align the same features across different human faces. The faces can then be segmented and mapped onto common canonical planar domains for subsequent registration and comparison, as shown in Figure 6 (d) and (e).

4.2. Brain Cortex Analysis. Another example is brain cortex comparison, we locate feature curves segmenting the whole surface into disks and annuli. These features are functional "landmarks" given by users. Our practical example is for medical imaging: The two cortex surface data are reconstructed using MRI images of one paralytic acquired at different times. The feature curves are manually labeled by the clinical doctor who is monitoring the recovery of this patient's brain. It is desirable to compare the cortex surfaces such that the corresponding curves and regions are matched. Noted here although these two brains are similar in terms of Riemannian metric, they cannot be matched simply via a rigid transformation. ${ }^{3}$

We apply our matching procedure as explained above. The feature curves for the first and the second brain are shown separately in Figure 7 (a) - (d) and (e) - (h) from different view directions. Feature curves and their corresponding signatures are shown in (m),(n) and (o),(p). By comparing their signatures, each curve on the first brain is mapped to the the curve on the second brain with the same color. The curves segment the cortex surfaces to four components, each of which is either a topological disk or an annulus; the segmentation is color encoded as shown in Figure 7 (a)-(h).

Each component on the cortex surface is conformally mapped to either the unit disk or the canonical planar annulus. Figure 7 (q) through (t) show the conformal mappings for the 4 components of the first cortex surface. Similarly, the components on the second cortex surface are conformally mapped to the unit disks or canonical annuli. By matching these canonical planar domains, the map between two cortex surfaces can be easily induced using existing method such as [10].

4.3. Elephant Gallop. We use an elephant gallop example to further evaluate our curve signatures and our surface comparison framework. As shown in Figure 8, there are two models of one elephant in different postures. Suppose we want to

\footnotetext{
${ }^{3}$ Because the cortex surfaces are highly convoluted, two points on the surface with small Euclidean distance in $\mathbb{R}^{3}$ may have huge geodesic distance on the surface.
} 


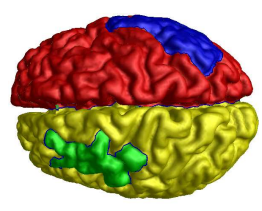

(a) First brain view from left

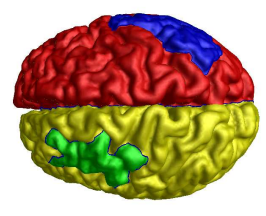

(e) Second brain view from left

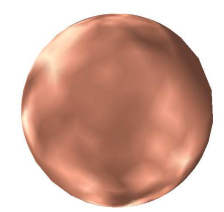

(i) Green Patch conformally mapped to disk

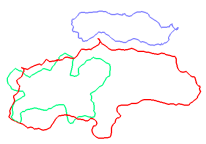

(m) Three curves on first brain

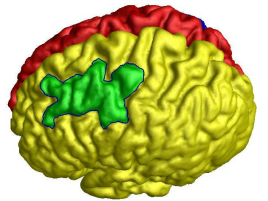

(b) First brain view from top

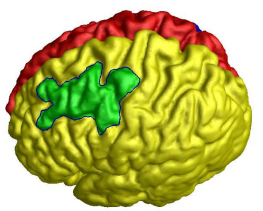

(f) Second brain view from top

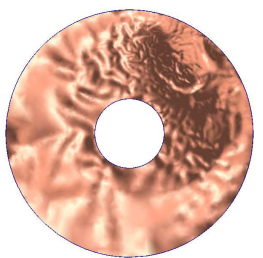

(j) Yellow Patch conformally mapped to annulus

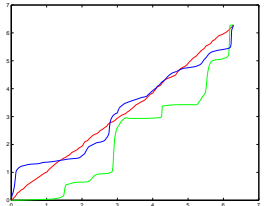

(n) Signatures of curves on 1st brain

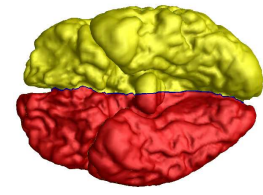

(c) First brain view from bottom

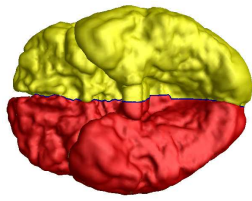

(g) Second brain view from bottom

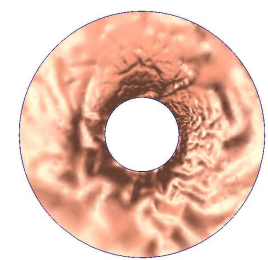

(k) Red Patch conformally mapped to annulus

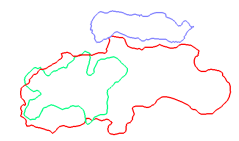

(o) Three curves on second brain

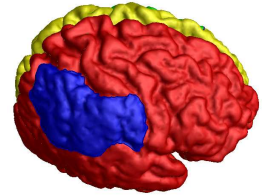

(d) First brain view from right

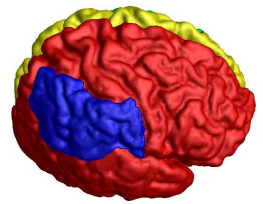

(h) Second brain view from right

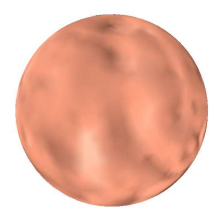

(1) Blue Patch conformally mapped to disk

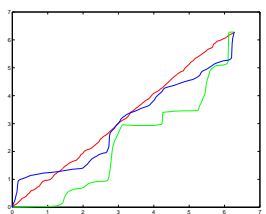

(p) Signatures of curves on 2nd brain

FIG. 7. Surface match on brains using curve features similarity comparison.

compare these two models, we first label feature curves which segment the elephants into several parts. We compute signatures for all curves on both surfaces, as shown in the third row. Every signature of curve on one surface is matched to the most similar signature of curve on another surface. The matched pairs are all placed in the same column, and each feature curve and its corresponding signature are drawn in the same color. The experiment results demonstrate that the correct matching can be induced automatically without human intervention. This attractive property on 


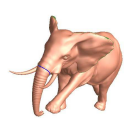

(1) Nose

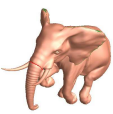

(7) Nose

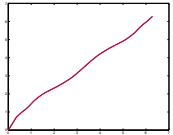

$(13)$

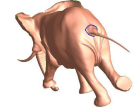

(2) Tail

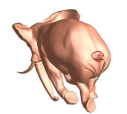

(8) Tail

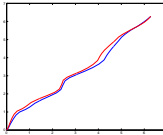

(14)

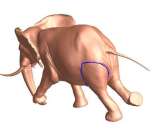

(3) Leg 1

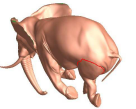

(9) Leg 1

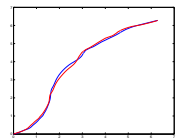

$(15)$

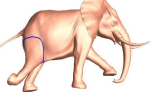

(4) Leg 2

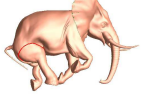

(10) Leg 2

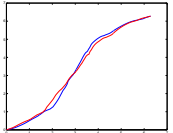

(16)

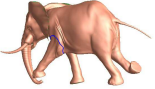

(5) Leg 3
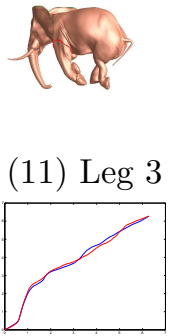

(17)

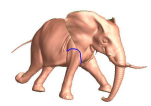

(6) Leg 4

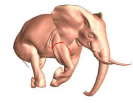

(12) $\operatorname{Leg} 4$

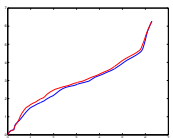

(18)

FIG. 8. Feature curves and their signatures on two elephant models with different postures. The first row (1)-(6) shows one geometric configuration of the elephant from different view directions; the second row (7)-(12) shows another model from different view directions; the third row (13)(18) shows the signatures. Note that, each column shows a special curve on two models and their signatures, and the curve is depicted with the same color as its signature. In (13), the red and blue signatures are almost identical so that they overlap and are not distinguishable.

curve signatures results from the fact that the signatures for corresponding curves are very similar, and the underlying reason of this fact is that the skin deformation is very close to isometry because the stretch of skin under these kinds of deformation is relatively small.

Once the corresponding feature curves are matched, the surfaces are segmented into several parts with explicit correspondence established by the segmenting curves. These parts are then considered separately on their own canonical planar domains, as shown in Figure 9. On each domain, we use the stretching and bending functions to compare their differences. The conformal factor and mean curvature are computed and colorized in the original surface to show the function value distributions. We color-code the conformal factor of the first model in the first row, (Figure 9 (1)-(8)), and color-code this model's mean curvature in the second row ((9)-(16)); similarly we color-code the conformal factor and the mean curvature of the second pose in the third ((17)-(24)) and fourth $\operatorname{rows}((25)-(32))$. The matching difference between two surfaces based on the functions on 2D domains are color-coded on the first pose and shown in the last row ((33)-(40)). The color-code scheme is the same as in the previous example (Figure 5 (c)), where "red" represents the max value and "blue" is for the min value. Note that, the last column is color coded in one uniform scheme. And the results shows that largest stretching and bending differences locate on leg 


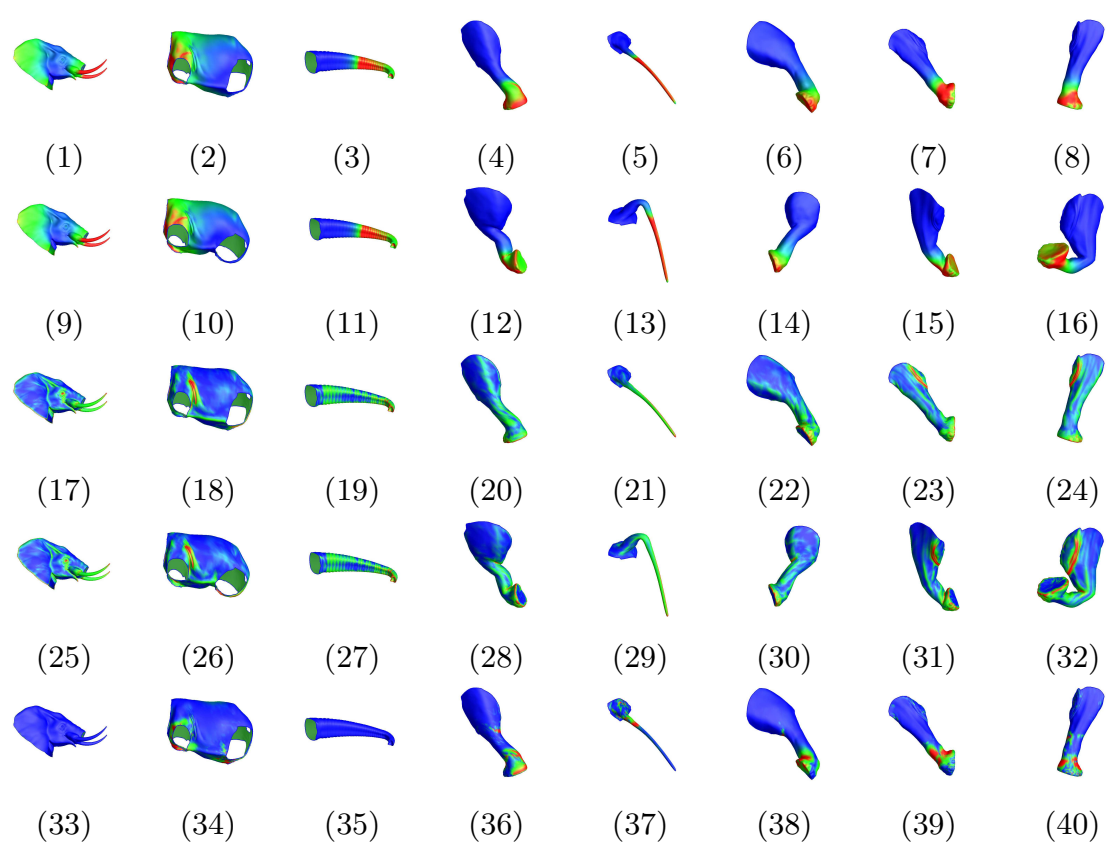

FIG. 9. Segmented parts from the elephant model and their color-coded function distributions, highlighting their intrinsic differences. The first row (1)-(8) show the conformal factor function distributions mapped on the original surface of the first model; the second row (9)-(16) show the conformal factor distributions of the second model; the third row (17)-(24) shows the mean curvature distributions of the first model; the fourth row (25)-(32) shows the mean curvature distributions of the second model; the last row (33)-(40) shows the difference of conformal representation between two models.

joints and ankles.

4.4. The Collapsing Horse. The next experiment is to compare a horse and its collapsed pose. Users first mark feature curves on one pose. With their signatures, we could reconstruct the curves on the second surface. Techniques introduced in [27] can be used to reconstruct the curve on the complex domain, which corresponds to a unique curve on the spherical domain. Combined with three predefined markers introduced in section 2.5 and the mapping from the original surface to the sphere, the unique curve on the original surface can be reconstructed. With this process, feature curves can be transferred onto the second object as shown in Figure 10. The original feature curves on rest pose, their signatures, and the transferred curves are shown in the three rows in Figure 10 respectively.

The conformal factor and the mean curvature distributions of all parts are computed and color-coded in the first four rows of Fig 11 (the first two rows are for the standing pose, while the third and fourth rows are for the collapsed pose).

The surface comparison framework can be interactively controlled by changing 

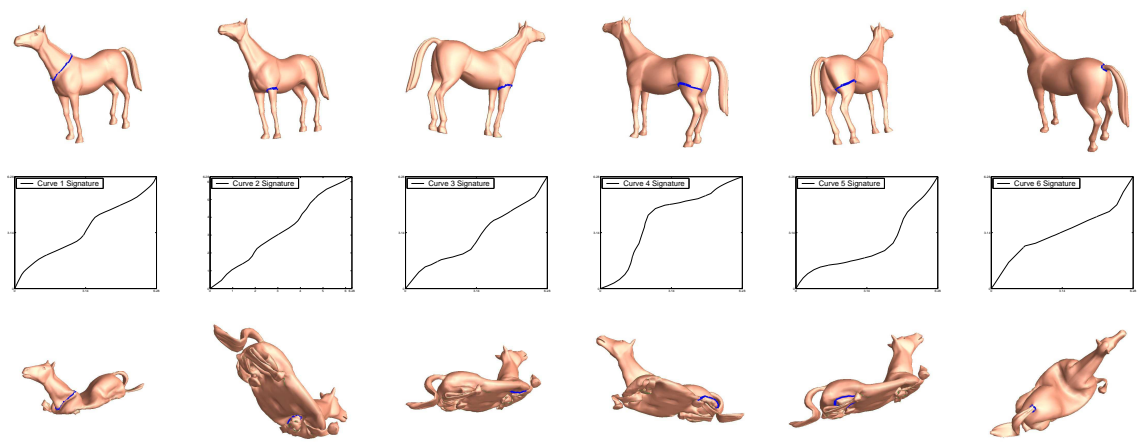

FIG. 10. The first row views feature curves on the standing-horse model; the second row shows their corresponding signatures; the third row displays the accordingly reconstructed curves on the collapsed-horse model.

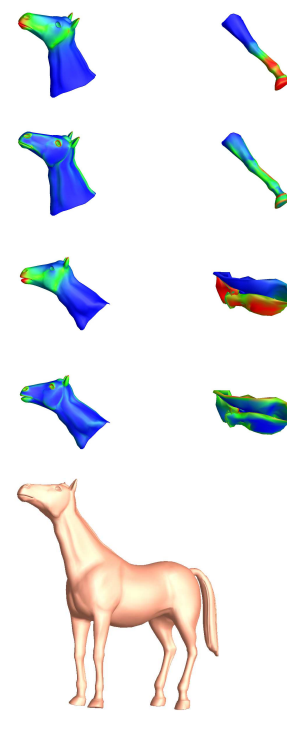

(a)
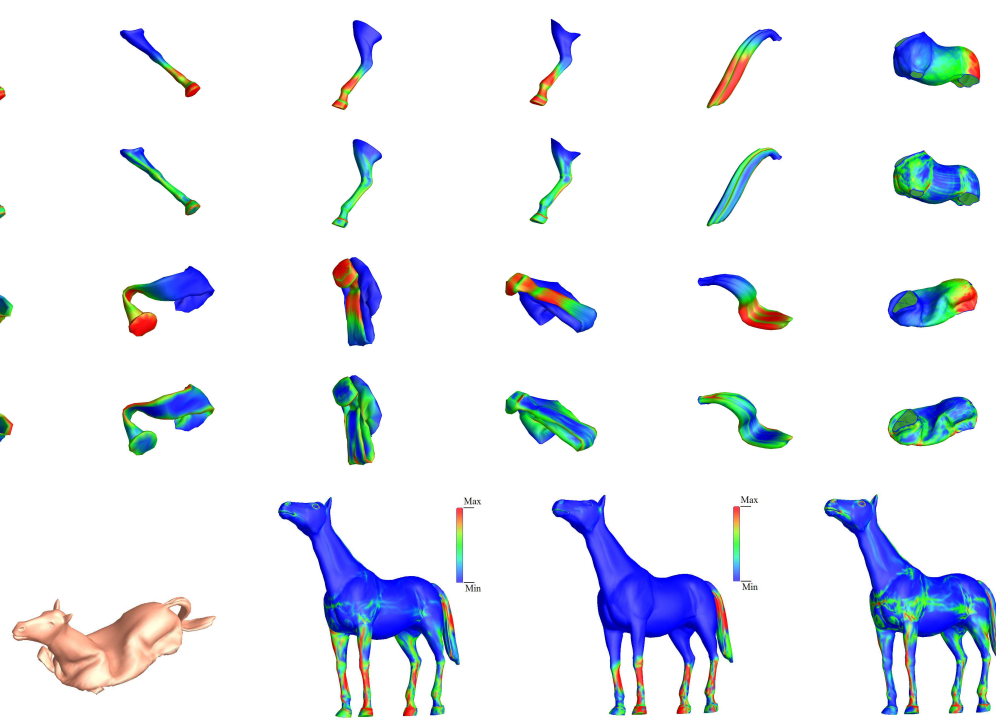

(b)

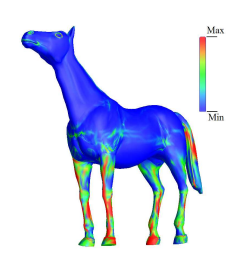

$(c)$

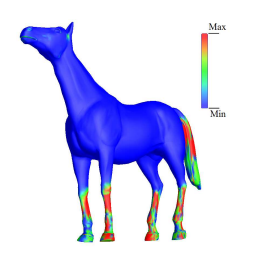

$(d)$

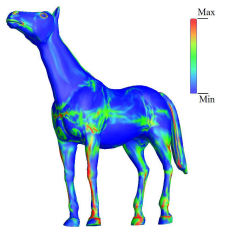

$(e)$

FIG. 11. The first and the second row color-code conformal factor $\lambda$ and mean curvature $H$ of the standing horse model; the third and fourth rows are of the collapsed horse model; the last row shows the final matching results between the standing model (a) and the collapsed model $(b)$, with $(c)-(e)$ color-coding differences on conformal representation, $\lambda$, and $H$ respectively. (Mesh size: 17k Triangles)

weights of the two terms in our matching energy. For example, if isometry-invariant comparison is preferred, only stretching factor needs to be considered. So by ignoring the mean curvature, a metric invariant under bending is designed, which naturally leads to a bend-invariant or pose-invariant result. The conformal representation difference between the two horse models $(a)$ and $(b)$ is color-coded on the first model as shown in Figure $11(c)$ and the difference ignoring the bending term is shown in 
Figure $11(d)$; also, the difference with only the bending term is color-coded in $(e)$. As shown in the above examples, our matching algorithm finds out between two complicated objects a difference distribution which can be flexibly adjusted for different goals such as the bending-invariant purpose. Since it can catch the difference on the metric ignoring the embedding of the surface in $\mathbb{R}^{3}$, it becomes a useful tool for non-rigid matching applications. One example is the colons matching and analysis in medical imaging. People with different poses under CT scans might have large bending differences on their colons with little changes in metric, in which case such a bending-invariant matching is ideal for the analysis purpose.

5. Conclusions and Future Work. We have designed a metric space for simple closed curves on genus-zero surfaces via conformal mappings. Curves on surfaces are represented by equivalence classes of diffeomorphisms of the unit circle to itself. The proposed curve signature corresponds uniquely to the curve defined on a surface. It includes information of how the curve segments the surface, which are invariant under isometry and stable under near-isometric transformation of surfaces. Therefore, the signature enables a powerful practical tool for the effective analysis of curves and surfaces among geometrically similar objects.

Besides the above theoretical results, we develop a framework for shape registration and comparison guided by feature curves alignments. After curves with the most similar signatures are correctly identified and aligned, genus-zero surfaces are then segmented into several parts and registered separately. This automatic process accurately forces the alignment of feature curves and alleviates the difficulties of 3D surface matching by reducing it to the simple comparison of functions defined on canonical planar domains. Also, the algorithm can be flexibly adjusted to provide a pose-invariant shape descriptor.

One potential limitation is that the curve signature developed in this paper is perhaps best suitable to analyze curves defined on one surface or two surfaces of similar geometry. When the signature is compared for curves defined on surfaces with large difference, it is only stable when there exists a near-isometric mapping between the surfaces. In general, aligning curves defined on surfaces with dramatically different geometry is technically challenging.

Constructing shape space of curves on surfaces with arbitrary topology is promising and challenging. We plan to explore further along these directions in the near future.

\section{REFERENCES}

[1] M. Ankerst, G. Kastenmüller, H.-P. Kriegel, and T. Seidl, 3d shape histograms for similarity search and classification in spatial databases, in: SSD, pages 207-226, 1999. 
[2] R. Basri, L. Costa, D. Geiger, and D. Jacobs, Determining the similarity of deformable shapes, 38(1998), pp. 2365-2385.

[3] S. Belongie, J. Malik, And J. Puzicha, Shape context: A new descriptor for shape matching and object recognition, in: NIPS, pages 831-837, 2000.

[4] S. Biasotti, S. Marini, M. Mortara, G. Patanè, M. Spagnuolo, and B. Falcidieno, $3 D$ shape matching through topological structures, in: DGCI, volume LNCS 2886, pages 194203. Springer-Verlag, 2003.

[5] I. Cohen, N. Ayache, And P. Sulger, Tracking points on deformable objects using curvature information, in: ECCV, pages 458-466, 1992.

[6] T. K. Dey, J. Giesen, And S. Goswami, Shape segmentation and matching with flow discretization, in: WADS, pages 25-36, 2003.

[7] M. Frenkel And R. BASRI, Curve matching using the fast marching method, in: EMMCVPR, pages 35-51, 2003.

[8] W. Fulton, Algebraic Topology. Springer-Verlag, 1995.

[9] R. Gal, A. Shamir, and D. Cohen-Or, Pose oblivious shape signature, 2005.

[10] X. Gu And B. C. VemurI, Matching 3d shapes using 2d conformal representations, in MICCAI (1), pages 771-780, 2004

[11] X. Gu, Y. Wang, T. Chan, P. Thompson, and S.-T. Yau, Genus zero surface conformal mapping and its application to brain surface mapping, in: IPMI, 2003.

[12] X. Gu And S.-T. Yau, Global conformal surface parameterization, in: Proc. Eurographics/ACM SIGGRAPH Symp. Geometry Processing, pages 127-137, 2003.

[13] X. Gu AND S.-T. YAU, Surface classification using conformal structures, in: ICCV, pages 701-708, 2003.

[14] M. Hilaga, Y. Shinagawa, T. Kohmura, and T. L. Kunit, Topology matching for fully automatic similarity estimation of 3d shapes, in: SIGGRAPH, pages 203-212, 2001.

[15] E. Hildreth, The Measurement of Visual Motion, MIT Press, Cambridge, 1983.

[16] M. Kazhdan and T. Funkhouser, Harmonic 3d shape matching, in SIGGRAPH 2002 Technical Sketch, 2002.

[17] M. M. Kazhdan, B. Chazelle, D. P. Dobkin, T. A. Funkhouser, and S. Rusinkiewicz, A reflective symmetry descriptor for 3d models, Algorithmica, 38:1(2003), pp. 201-225.

[18] J. W. Milnor, Morse Theory, Princeton Univ. Press, 1963.

[19] G. Mori, S. Belongie, And J. Malik, Efficient shape matching using shape contexts, IEEE Transactions on Pattern Analysis and Machine Intelligence, 27:11(2005), pp. 1832-1837.

[20] M. Novotni And R. Klein, 3d zernike descriptors for content based shape retrieval, in: SPM, pages $216-225,2003$.

[21] R. Ohbuchi, T. Otagiri, M. Ibato, and T. Takei, Shape-similarity search of threedimensional models using parameterized statistics, in: PG, pages 265-275, 2002.

[22] R. Osada, T. A. Funkhouser, B. Chazelle, and D. P. Dobkin, Matching $3 d$ models with shape distributions, in: SMI, pages 154-166, 2001.

[23] A. Pentland and S. Sclaroff, Closed-form solutions for physically based shape modeling and recognition, IEEE Trans. Pattern Anal. Mach. Intell., 13:7(1991), pp. 715-729.

[24] M. Reuter, F.-E. Wolter, And N. Peinecke, Laplace-spectra as fingerprints for shape matching, in: SPM, pages 101-106, New York, NY, USA, 2005. ACM Press.

[25] D. Saupe And D. V. VRanic, 3d model retrieval with spherical harmonics and moments, in: Proceedings of the 23rd DAGM-Symposium on Pattern Recognition, pages 392-397, London, UK, 2001. Springer-Verlag.

[26] T. B. Sebastian, P. N. Klein, And B. B. Kimia, On aligning curves, PAMi, 25:1(2003), pp. $116-125$. 
[27] E. Sharon and D. Mumford, 2d-shape analysis using conformal mapping, in: CVPR (2), pages 350-357, 2004.

[28] K. Siddiqi, A. Shokoufandeh, S. J. Dickinson, And S. W. Zucker, Shock graphs and shape matching, Int. J. Comput. Vision, 35:1(1999), pp. 13-32.

[29] H. Sundar, D. Silver, N. Gagvani, And S. J. Dickinson, Skeleton based shape matching and retrieval, in: SMI, pages 130-142, 290, 2003.

[30] H. D. TAGARE, Shape-based nonrigid correspondence with application to heart motion analysis, in: IEEE Trans. Medical Imaging, 18(1999), pp. 570-578.

[31] M. Taniguchi, An Introduction to Teichmuller Spaces, Springer, 1992.

[32] L. Younes, Computable elastic distances between shapes, SIAM J. Appl. Math., 58:2(1998), pp. $565-586$. 